\title{
Pratiques
}

Linguistique, littérature, didactique

183-184| 2019

oralité, littératie

\section{« On lui acheta un cartable et des sandales »}

Une ethnocritique du roman de Malika Mokeddem Les Hommes qui marchent

Simon Lanot

\section{(2) OpenEdition}

\section{Journals}

Édition électronique

URL : http://journals.openedition.org/pratiques/7253

DOI : $10.4000 /$ pratiques.7253

ISSN : 2425-2042

Éditeur

Centre de recherche sur les médiations (CREM)

Référence électronique

Simon Lanot, « "On lui acheta un cartable et des sandales » », Pratiques [En ligne], 183-184 | 2019, mis en ligne le 30 décembre 2019, consulté le 11 octobre 2020. URL : http://journals.openedition.org/ pratiques/7253; DOI : https://doi.org/10.4000/pratiques.7253

Ce document a été généré automatiquement le 11 octobre 2020

(C) Tous droits réservés 


\section{«On lui acheta un cartable et des sandales »}

Une ethnocritique du roman de Malika Mokeddem Les Hommes qui marchent

Simon Lanot

Ce qui nous manque est inscrit dans la mélodie de chaque pas

(Hawad, 1985)

Le roman Les Hommes qui marchent, publié en 1990, met en scène l'entrée dans la culture écrite de L.Ajalli, une jeune Algérienne au tournant de la colonisation et de l'indépendance, et aboutit à son passage à l'état d'écrivain. Le roman est un hommage aux ancêtres nomades, les " hommes qui marchent ", et se caractérise par un retour de l'oralité dans la littératie la plus valorisée : la littérature ${ }^{1}$. Le roman est un tombeau pour les oralités dont le personnage de Zohra, la grand-mère de Leïla, serait le dernier représentant : cette figure d'aïeule est, selon la terminologie de D. Fabre, un « individumonde "; elle est à la fois un individu singulier et une nomade emblématique, dont le « destin individuel reflète, concentre et achève » une " histoire commune ${ }^{2}$ ». L'éloge d'un passé révolu caractérisé par l'oralité et le nomadisme est le moyen, dans le roman, de pointer les travers de la société algérienne moderne, pétrifiée par une idéologie rétrograde qui a oublié les valeurs anciennes et trahi les aspirations progressistes portées par l'indépendance. Donc l'oralité et la littératie apparaissent comme deux faces d'une certaine conception de la liberté sous le signe du nomadisme, tandis que la sédentarité charrie tout l'échec d'une révolution qui a renié ses racines et ses espérances.

\section{Un T au Sahara : ethnocritique et ethnographie touarègue}

2 L'ethnocritique de la littérature, qui est la démarche que nous suivrons, est une herméneutique qui part de l'hypothèse que « les traits de culture présents dans l'œuvre 
littéraire (qu'on pourrait nommer "culturèmes") s'organisent en systèmes discursifs et en cosmologies culturelles, toujours métissées et plurielles ${ }^{3} »$. Ainsi nous étudierons "cette dialogisation, au sein de l'œuvre, d'univers symboliques plus ou moins hétérogènes et hybrides ${ }^{4} »$. La pluralité des cultures traverse et enrichit le personnage ainsi que l'écriture du roman; or cette pluralité se fissure dans un contexte de guerre civile accompagnée par une déchirure entre les communautés juives, arabes, berbères et européennes qui composent un pays en profonde mutation. Leïla, le personnage principal, est initiée à la littératie grâce à la scolarisation; dans cette étude, le texte sera analysé comme le récit de l'entrée d'une jeune fille dans la culture écrite, en partant de l'hypothèse de l'ethnocritique d'une homologie entre rite de passage et récit.

3 Les dichotomies structurant l'idéologie sur laquelle s'est fondée l'indépendance (colons/indigènes, Arabes/Berbères, musulmans/non musulmans) ${ }^{5}$ sont déconstruits par le roman, dans lequel est repensé de manière plus complexe, plus humaine aussi, l'identité «en marche ${ }^{6}$ » de sociétés vivant dans un espace géographique unique, le Sahara, pris dans la tourmente de la colonisation puis de la révolution algérienne. Le texte repense les fractures sociales et culturelles en dépassant les dichotomies simplistes et mortifères. L'oralité se confronte aux cultures arabes, maures, juives et berbères, à cette diversité culturelle niée par le pouvoir mis en place après l'indépendance. L'écriture, qui se présente comme retranscription des rites, des coutumes et d'une cosmologie portée par des voix venues d'outre-tombe, participe à la re-légitimation des cultures orales: en retour, l'écriture se pose comme une réelle continuité culturelle de l'oralité, à l'inverse des idéologies meurtrières (colonialisme, FLN, islamisme), qui ont trahi les héritages culturels.

\section{De la saga à la sédition}

Le roman est un métier à tisser sur lequel se nouent trois fils : une saga familiale, un récit initiatique et une chronique historique. La saga familiale est un véritable conte généalogique, racontée en guise d'héritage par Zohra, la grand-mère de Leïla : l'écrit se fait ici invocation, imitant l'oralité perdue. Zohra raconte comment le clan des Ajalli a été contraint de se sédentariser et de se fixer dans la ville saharienne de Kenadsa. Le récit initiatique est un roman de formation, qui raconte l'entrée dans la littératie de Leïla, et ses stratégies d'émancipation contre tous les systèmes coercitifs : contre la colonisation qui la relègue au rang d'indigène, contre le clan familial qui la contraint à épouser son cousin dès la puberté, puis contre l'État algérien qui borne ses exigences de liberté. Au terme du récit, la jeune fille subvertit les logiques liberticides pour passer non au statut d'épouse comme l'attend le clan, mais à celui de femme de lettres. Enfin, le roman est une chronique de la révolution algérienne, de sa lutte pour l'indépendance à la mise en œuvre de grandes réformes politiques et sociales : l'écrit prend tantôt la forme de l'analyse ethnographique (en décrivant les diverses communautés au sein de la société algérienne), tantôt celle de la chronique subjective des années 1950-1970 ; le discours politique est marqué par une forte tonalité polémique.

5 Un étrange motif (un motif étranger ?) apparaît quatre fois au cours du texte : le passage des «hommes bleus» (Mokeddem, 1990, p. 113). Cette appellation désigne les Touaregs ${ }^{7}$, nomadisant entre l'Algérie, la Libye, le Mali et le Niger. Le passage d'une « tribu berbère » (ibid., p. 113) à Kenadsa est mis en scène dans les dans les chapitres V, XI, XIII et XIV. Chaque fois, la tribu ne reste que le temps d'une soirée près de la maison 
familiale, et l'épisode n'occupe que deux pages. Chronologiquement, les «hommes bleus » passent au moment de la grève générale de janvier 1957, au moment de la proclamation de l'indépendance en juillet 1962, en novembre 1965 après le coup d'État de H. Boumédiène, puis peu après la mort de Zohra et l'obtention du bac de Leïla (1967). L'analyse de ces quatre "passages" dans le roman est, selon nous, le moyen de pénétrer la logique poétique du texte et d'appréhender son sens éthique et politique. Ils marquent un contraste important avec l'ordre narratif qui voit l'émergence d'une nation moderne en voie d'indépendance : l'ordre nomade berbère va à rebours de la construction de la nation algérienne; son organisation sociale, sa cosmologie et sa pensée vont à l'encontre de l'ordre littératien qui s'étend avec la modernité. Leur mode de vie entre en écho avec la voix de Zohra : «surgis du passé de Zohra ( (ibid., p. 113), ils sont symboliquement le relai dans le présent du passé familial auquel Leïla a accès via les contes de sa grand-mère.

\section{Tisser les voix ${ }^{8}$ des cultures orales : pénétration de la pensée sauvage dans la raison graphique}

6 Le roman commence dans le milieu des Maures nomades du Sahara. À plusieurs reprises, le texte désigne la famille Ajalli comme des Bédouins, c'est-à-dire des nomades arabophones qui nomadisent dans l'Ouest du Sahara, entre l'actuelle Algérie et la Mauritanie (ibid., p. 14). Pour les historiens et les anthropologues, les Maures descendent des Berbères Sanhadja, nomades sahariens dont les hommes portaient le litham (ou le tagelmust en tamasheq), c'est-à-dire le voile de bouche : c'est entre le xve et le XVII siècle, selon $\mathrm{H}$. Claudot-Hawad, que "les hommes voilés se scindent progressivement en deux ensembles distincts : d'un côté les Maures devenus "arabes" et de l'autre les Touaregs demeurés "berbères" "». Les Maures s'arabisent progressivement à partir du XIV siècle, avec l'arrivée au Sahara d'Arabes descendant d'une tribu yéménite : les Bani Hassân. Cette invasion a causé, selon C. Taine-Cheikh, un "phénomène de substitution linguistique " caractérisé par «la diffusion de l'arabe comme langue usuelle et populaire de communication ${ }^{10} »$. De ce fait, la langue utilisée par les Ajalli est l'arabe dialectal.

7 C'est donc l'oralité qui domine, dans la société maure. Selon V. Cnockaert et S. Dumoulin, « l'oralité renvoie à tout ce qui se rattache à une culture orale, c'est-à-dire une culture fondée sur un régime de vie antérieur ou extérieur à l'empire de l'écrit, une culture qui repose principalement sur des rites coutumiers, des pratiques rituelles ${ }^{11}$ ». Les Ajalli n'ont pas appris à lire, ils ne savent déchiffrer ni les tifinagh de leurs voisins Touaregs, ni l'alphabet arabe qui est pourtant présent parmi eux dans les exemplaires du Coran et les talismans. En suivant la distinction de J.-M. Schaeffer, nous pouvons dire qu'il ne s'agit pas d'une "oralité primaire" (une société qui ignore totalement l'écriture), mais d'une « oralité secondaire ${ }^{12} »$ : les traditions orales dominent dans une société qui connaît l'écriture (qui la considère comme sacrée ici) mais qui ne la manipule pas. L'écriture y est présente sous deux formes : d'une part, l'écrit sacralisé (les talismans et les sourates du Coran, lettres calligraphiées que l'on porte sur le corps), d'autre part, «l'écrit oralisé » que C. Taine-Cheikh appelle l'aurai; elle définit l'auralité comme un "ordre discursif qui transcende l'opposition oral/écrit et met l'accent sur l'aurai ${ }^{13} »$. Le Coran fait l'objet d'une récitation: il n'est pas déchiffré mais récité, psalmodié, intégré et restitué corporellement par la voix et le balancement du 
corps. L'écrit coranique (l'écrit de Dieu lu à voix haute par le Prophète) et le hadith (paroles du Prophètes mis par écrit par ses disciples) peuvent se passer de la compétence de la lecture. Les Ajalli disent :

«Lire et écrire? Au sein du monde de l'oralité, pure extravagance. Depuis des siècles, personne n'avait eu recours à l'écriture. Le Coran, on en savait juste les versets indispensables aux prières. Nos mœurs étaient empreintes du hadith. Notre histoire ne se couche pas entre l'encre et le papier. Elle fouille sans cesse nos mémoires et habite nos voix...» (ibid., p. 16)

8 L'écrit prend place dans les représentations cosmiques et sociales et dans les pratiques techno-économiques. Ainsi, lorsque Djelloul, vers 1840, décide d'apprendre à lire et de devenir taleb (étudiant), le clan des Ajalli y voit l'occasion d'une augmentation de leur prestige social ainsi qu'une manne économique : «Un homme sachant lire et écrire le Coran - qu'avait-on d'autre à lire ? - pouvait même être très utile. Il les protégerait contre le mauvais oeil et les maléfices des démons. Sans oublier les gains obtenus par les demandes de talismans » (ibid., p. 17). L'écriture, qui est nécessairement l'écriture $d u$ Coran, dans cette cosmologie, est sacrée et possède la vertu de protéger le clan du malheur. Passant du «niveau cosmologique » au «niveau techno-économique ${ }^{14}$ », la pratique de l'écriture est considérée comme un moyen de revenus conséquents puisqu'elle est un art, une teknè, produisant des objets pouvant être vendus chers, compte-tenu du prix qui leur est accordé au niveau cosmologique ${ }^{15}$. L'Islam modifie donc la pure oralité antérieure ou extérieure à l'empire de l'écrit, puisque l'écriture acquiert un prestige social et gain économique en conséquence du crédit de sacralité qui lui est conféré. Seule l'écriture religieuse et inscrite en lettres arabes occupe alors une place: l'écriture en alphabet latin ne jouit d'aucun prestige, et la littérature profane est considérée comme suspecte. Or c'est justement cette dimension païenne de la littérature arabe qui attire Djelloul ; au lieu de lire le Coran, il lit la poésie de la Jahiliya (littéralement de l'ère de l'ignorance) datant d'avant l'Hégire. Loin de se limiter au caractère magique de la lettre matérielle, Djelloul est attiré par le «monde inconnu » que lui révèle le contenu de l'écriture.

9 La pure oralité ne subsiste, dans le milieu maure, que dans « la bouche du griot ${ }^{16}$ ». Aussi peut-on considérer l'aïeule Zohra comme une griote, celle qui maîtrise la parole et dont la réputation de sagesse se répand sur les routes des caravanes. Pourquoi les « hommes bleus» font-ils un "détour de cent kilomètres» (ibid., p. 237) pour rendre visite à Zohra? H. Claudot-Hawad insiste sur le fait que la "poésie demeure une passion nomade. Pour écouter un poète ou un chanteur réputé, les Touaregs sont prêts à parcourir des centaines de kilomètres ${ }^{17} »$ : c'est bien ce qui se passe dans le roman. Zohra se distingue en effet par sa capacité à produire des tropes poétiques, et à tenir une conversation subtile. Pour les Touaregs, l'intelligence est la vertu supérieure, laquelle consiste à produire ou comprendre des iggitan ; J. Drouin définit l'iggi (pluriel : iggitan) comme un "discours sémantiquement indirect qui n'est pas directement compréhensible ${ }^{18}$ ». Le sens de la répartie s'évalue à la pertinence de l'iggi; ainsi, lorsque Zohra reprend ses petits-neveux tenant des propos racistes contre les Noirs, elle lui rétorque : «si tu n'aimes pas le noir, mon petit, tu n'as qu'à l'enlever de tes yeux » (ibid., p. 161). Elle produit ici un iggi (qui n'est pas sans rappeler certaines saillies des Évangiles), plein de subtilité et de sagesse : elle apprend ainsi aux enfants qu'ils ont pour ancêtre une servante sénégalaise, et démontre l'inanité du racisme dans le Sahara, terre d'échanges et de mélanges, plus efficacement que par des discours. Le trait d'esprit mêle création verbale et sagesse; les paroles poétiques de Zohra, dites ou 
chantées, prennent une dimension prophétique pour Leïla : "les pieds ont beau courir, le regard ira toujours plus loin qu'eux, quoi qu'ils fassent... Ils ne peuvent emprisonner ni la volonté de liberté, ni l'espoir d'y parvenir." Leïla, habituée aux métaphores de l'aïeule, ne comprit pas complètement celle-ci » (ibid., p. 173). Le texte emploie le mot métaphore qui ne rend qu'imparfaitement la trope (l'iggi) de la griote. Le mot griote (vraisemblablement d'origine portugaise) n'est pas employé : les Touaregs qui ont parcouru des centaines de kilomètres pour l'écouter l'appellent, dans la langue de leur hôte, «la cheikha ». Le choix de ce mot arabe qui connote davantage la sagesse que la subtilité verbale doit traduire ce qu'en tamasheq on nomme une taggagt : figure de passeur servant "d'intermédiaire entre les parties jumelles de l'univers, entre le visible et l'invisible, entre le connu et l'inconnu ${ }^{19}$ ». Zohra est une figure assurant le passage, la transmission entre les univers culturels arabes, berbères et sud-sahariens qui traversent le Sahara des hommes qui marchent; pour présenter le personnage, la narratrice écrit que "Zohra était le désert» (ibid., p. 11) : il faut comprendre que sa parole est celle qui est propre à une géographie géologique et cosmologique qui échappe aux découpages imposés par la raison graphique des colonisateurs et des États post-coloniaux.

\section{Espace et labyrinthes ${ }^{20}$ : le pays, la carte et les archives}

10 Tout le premier chapitre raconte la saga de la famille Ajalli, dans un milieu et une époque qui ignore le découpage du temps et de l'espace tel que le conçoit la raison graphique. Le Sahara des récits de Zohra est ce que M. Bakhtine appelle un chronotope, qui obéit à sa propre logique spatio-temporelle, économique et cosmologique. Les Ajalli nomadisent dans un espace avant tout physique, géologique, qui considère le Sahara comme un ensemble cohérent, dont les frontières sont davantage géologiques et ethniques que politiques. Ainsi, la frontière des transhumances des Ajalli n'est pas celles imposées par l'occupation turque puis par la colonisation française, mais par la géologie : le « Tell, toujours malsain. Des roumis y ont remplacé les Turcs » (ibid., p. 17). Le récit de Zohra à ses descendants devenus sédentaires et retraçant ce qui a eu lieu un siècle auparavant, s'énonce dans les années 1950. Le texte place le lecteur dans la situation des auditeurs, lesquels étant nés dans une colonie française - l'Algérie -, pensent avec les repères hérités de la colonisation, ce qui les incite à poser la question de la datation. Ce à quoi répond Zohra : "Servitude de sédentaires que cette préoccupation!» (ibid., p. 10). La temporalité n'est pas affaire de dates mais un rapport particulier du nomade avec l'espace désertique: «Configuration d'éternité qui rend caduques les durées. Temps d'une marche. Temps d'une douleur, d'une rencontre. Temps d'une pluie, renaissance de la terre » (ibid., p. 10). À la temporalité linéaire de la raison graphique s'oppose la temporalité cyclique du milieu nomade que porte la voix de Zohra.

11 La raison graphique se veut une « mise en ordre graphique de l'intelligence du monde ${ }^{21}$

" : la cartographie du monde politique qui dessine les frontières à grands coups de traits aussi rectilignes qu'arbitraires retrace moins l'intelligence (la compréhension) du monde tel qu'il s'organise géologiquement et humainement, que sa mise en pièces par des accapareurs s'arrogeant les prérogatives de l'État. La colonisation, qui avait réprimé durement les « hommes bleus », laisse place à une décolonisation qui fixe des frontières 
plus rigides encore, annonciatrices de nouvelles entraves et persécutions pour les nomades peuls, maures et touaregs. Ainsi, lorsque les « hommes bleus » viennent chez Zohra pour célébrer «el Houria» (littéralement la liberté, l'indépendance) en 1962, cette libération limitée à la création de nouveaux États prend aussitôt une forme carcérale : "Ils dirent qu'ils avaient fui les fouilles et les barbelés vers le Mali et le Niger » (ibid., p. 237).

Le roman, qui racontera un moment important de l'Histoire de l'Europe et de l'Afrique, commence donc dans un chronotope an-historique, c'est-à-dire hors des conceptions de l'histoire selon la pensée domestiquée, puisque la littératie n'y a pas (encore) cours. Sans littératie, il ne peut y avoir de vision historique au sens d'une chronologie, d'une liste d'événements notés. En effet, comme le démontre J. Goody, les "archives sont une condition préalable de l'Histoire ${ }^{22} »$. Zohra raconte l'histoire des ancêtres, en particulier la geste de Djelloul, le jeune homme qui, à partir de sa découverte de l'écriture, est devenu « Bouhaloufa », c'est-à-dire « l'homme au cochon ». Cet aïeul, qui vivait à l'époque des débuts de la colonisation («Des roumis ont remplacé les Turcs »), devient une figure mythique. Nous sommes donc dans une histoire mythique, laquelle est, selon C. Lévi-Strauss, disjointe et conjointe du présent : «Le passé disjoint du mythe s'articule, d'une part, avec la périodicité biologique et saisonnière, d'autre part, avec le passé conjoint qui unit, tout au long des générations, les morts et les vivants ${ }^{23}$ ». Le récit généalogique qui s'appuie sur le caractère concret des saisons («l'année de la très grande sécheresse », p. 10), tisse un lien privilégié avec les morts, ancêtres mythiques du passé, nécessairement disjoint puisque ces êtres étaient « d'une autre nature ${ }^{24}$ », mais également conjoint puisque le récit a pour fonction d'affirmer qu'il ne s'est rien passé de vraiment important depuis.

13 L'ancêtre Bouhaloufa est un être d'une autre nature, un être hybride du fait de sa pratique de la lecture et de l'écriture. Son nom coagule la figure sacrée du patriarche "Père " (Abou réduit à Bou servant de préfixe dans certains patronymes) - avec l'animal impur "cochon» (Halouf). Ce nom est composé d'un préfixe arabe (Bou-) et d'un radical berbère (halouf) passé en arabe dialectal, c'est-à-dire dans l'arabe parlé. Cet être mythique est le premier des Ajalli à avoir choisi de se sédentariser, suite à sa découverte de l'écriture. Jeune encore, il avait rencontré un Taleb qui pour la première fois l'avait mis en contact avec un livre : «le garçon n'avait jamais entendu parler de l'écriture » (ibid., p. 14). Dans ce milieu où domine l'oralité, l'écriture est une révélation parce que c'est une découverte visuelle avant d'être auditive. Le jeune garçon est frappé de stupeur par les caractères arabes qui figurent les récits des Mille-et-Une-Nuits: "Comment ces caractères inertes pouvaient-ils contenir tant d'histoire, d'intrigues, de combats et de beautés ? Dans la kheïma [...], Djelloul découvrit un monde de palais » (p. 14). La phrase est construite sur l'opposition tente (kheïma en arabe)/palais, désert/ ville, Sahara berbéro-maure/capitale abbasside. Quel est le "monde" inconnu que découvre le jeune garçon? Est-ce le Moyen-Orient arabo-persan ou le passé mythique de l'âge d'or du Calife Haroun-al-Rachid? Au-delà du dépaysement historicogéographique, le «monde » nouveau, c'est l'écriture elle-même. La véritable hybridité de Djelloul Bouhaloufa, c'est la fusion entre le parent et l'étranger, entre celui qui vient du monde de l'oralité et celui qui est entré dans le monde de la littératie. Littéralement, l'écriture aliène: «Djelloul se sentait devenir étranger aux siens. [...] Djelloul en prit conscience avec une joie douloureuse" (p.15). L'oxymore "joie douloureuse" synthétise la tension qui parcourt tout le roman. À la douleur de l'arrachement à la communauté s'agrège la joie de l'entrée dans un monde nouveau, l'écriture. Les récits et 
les contes de Zohra, à l'image du roman tout entier, matérialisent cette "joie douloureuse", réconciliant les antagonismes Arabe/Berbère, nomades/sédentaires, Europe/Maghreb, Maghreb/Afrique noire, à travers cette hybridation de la littératie avec l'oralité.

\section{Initiation à l'écriture : marginalisation, aliénation ou émancipation?}

14 L'ethnocritique pose l'hypothèse d'un rapport homologique entre rite et récit : certains récits littéraires fonctionnent comme un rite de passage. Selon A. Van Gennep, le rite de passage se déroule en trois phases : séparation, marge, agrégation ${ }^{25}$. Par exemple, pour passer à l'âge adulte et au statut de citoyen, comme le montre P. Vidal-Naquet (2005 [1981]) dans Le Chasseur noir, le jeune homme doit se séparer de la Cité et vivre, pour un temps, dans un espace marginal, ensauvagé, dans lequel les normes sont inversées, avant un nécessaire retour à l'ordre social dans lequel il convient de s'agréger. Dans Les Hommes qui marchent, le père de Leïla accepte de la scolariser, en hommage à Bouhaloufa, mais souhaite qu'elle se marie rapidement. Le récit fonctionne comme un rite de passage, dans lequel l'école, l'initiation scolaire, est la phase de marge, en attendant le retour à l'oralité, l'agrégation à la société nouvelle (l'Algérie indépendante), devant correspondre au passage à l'âge adulte qui doit conduire au statut d'épouse. L'initiation à l'écriture est donc un ensauvagement pour Leïla : l'ordre littératien vient inverser l'ordre coercitif du clan qui impose un ordre machiste. L'école et les livres sont un moyen d'échapper à l'ordre de la domus qui enferme les filles dans un rôle subalterne. Toute la stratégie de Leïla consiste à retarder au maximum le moment du retour et donc de la fin de la scolarité qui correspond à son mariage, nécessairement endogame et forcé. L'originalité du roman est de montrer que l'écriture, la culture du livre, est le lieu d'un ensauvagement qui l'arrache à l'ordre coercitif de la domus. Si la littératie est ce qui avait sédentarisé Bouhaloufa au siècle précédent, c'est la littératie qui rend Leïla nomade. Coincée entre la maison et l'école, c'est la lecture fait voyager l'écolière et lui donne accès à l'inconnu du monde : « Le livre n'était pas seulement un moyen d'évasion. Il était le complice, le soutien, l'enseignant. Il la structurait, la construisait. [...] Il était devenu le symbole de son refus du quotidien qu'on voulait lui imposer » (Mokeddem, 1990, p. 268).

Le roman relate l'initiation de Leïla, son entrée dans la littératie : le texte insiste sur les éléments concrets, matériels de la culture écrite. Le chapitre III raconte son entrée à l'école : "On lui acheta un cartable et des sandales. Sa mère lui confectionna une jolie robe. Et quand vint le grand jour, sa grand-mère l'accompagna à l'école » (ibid., p. 85). Cette première rentrée des classes est extrêmement ritualisée par le clan où domine l'oralité, car elle figure la première séparation et l'entrée dans la phase de marge. L'achat des sandales concomitante avec celui du cartable insiste sur la séparation, première phase du rite, phase dans laquelle la jeune fille quitte la domus. Séparée de ses parents, c'est Zohra qui l'accompagne jusqu'au seuil de l'école : la sheikha joue bien son rôle de passeur, d'intermédiaire entre les mondes et les âges. L'écriture est présentée d'abord par son aspect le plus matériel : «Plumes, cahiers et livres allaient devenir ses seules lignes de fuite hors de tous les enfermements » (ibid., p. 124). Ainsi, l'objet livre sert de rempart entre elle et la parole de sa mère, entre la parole injonctive et son oreille, qui semble se fermer tandis que son regard suit le fil du texte imprimé : « "Pose 
ce livre et fais ce que je te dis". [...] Au lieu de ranger le livre ouvert sur les genoux et d'obtempérer, Leïla le prenait à deux mains et le dressait contre son visage, entre elle et la donneuse d'ordre. [...] Les livres étaient devenus un refuge contre cette mère à laquelle elle ne voulait pas ressembler» (ibid., p.115). Leïla occupe une place de marginale chez elle; en cela elle suit la logique du rite dont la phase de marge se caractérise par l'inversion des normes sociales. Mais déjà elle subvertit le rite en transformant la phase dialectique de marge en une révolte durable contre l'ordre établi : les livres deviennent des "armes et moyens de résistance" (ibid., p. 124). La culture écrite modifie le corps de la jeune fille et le passage qu'elle est censée accomplir: refusant de vivre le même destin que sa mère qui multiplie les grossesses, Leïla devient anorexique tandis qu'elle " dévore » les livres (ibid., p. 268). Le "dressage littératien ${ }^{26}$ " modifie le corps de la jeune fille, tandis que le «capital littératien» et "l'habitus littératien ${ }^{27}$ » modifient en profondeur son comportement et sa pensée : "Craie, ardoise, encrier, plume, cahiers, livres... Leïla avait d'abord eu un contact charnel, sensuel, avec les éléments qui allaient façonner son esprit » (ibid., p. 124).

La littératie permet de subvertir l'ordre patriarcal et de détourner le sens du rite : au lieu d'un retour vers la société tribale, le rite que suit le récit est celui d'une agrégation au monde des lettres. Leïla refuse le retour pour choisir l'exil, c'est-à-dire l'installation durable dans la phase de la marge. Choisissant la langue française et la culture du livre, Leïla a conscience qu'elle devient étrangère pour le clan, mais qu'elle sera toujours l'étrangère, en situation de marginale, dans la société française. La fin du récit coïncide avec le refus du mariage, le refus du retour et de l'agrégation à la société algérienne et aux places qu'elle assigne aux femmes. Le roman se clôt sur une scène de retour au pays natal. Devenue une étrangère pour les Ajalli, Leïla ne revient pas pour s'agréger au clan et passer au statut d'épouse, mais pour passer au statut d'écrivain: elle entre dans l'écriture romanesque et commence la rédaction d'un premier roman, le roman que le lecteur achève justement. C'est en revenant au pays natal que Leïla choisit de rendre hommage à l'oralité dont elle est héritière; mais au lieu de reconnaître une dette à la génération de ses parents et à sa patrie, l'Algérie qu'elle a vu s'émanciper de la tutelle coloniale, elle veut retrouver le Sahara pré-colonial de ses ancêtres nomades, sans frontières nationales : elle veut retrouver l'oralité de Zohra, sa vision nomade de la vie. Donc, ce retour correspond, dans un premier temps, à une fuite-en-arrière dans l'oralité d'avant l'Histoire et la géographie telle que les conçoit la raison graphique. Dans un second temps, cette fuite-en-arrière correspond à une fuite-en-avant dans l'ordre littératien puisque Leïla fait de l'écriture sa profession, et qu'elle fait de sa vie un livre. Rêvant de l'ordre ancien, celui des « hommes qui marchent ", elle s'agrège à une autre société nomade, transfrontalière et transnationale, celle des écrivains. La nostalgie pour la vie nomade et la culture orale s'enracine dans le dépassement du présent déceptif par l'écriture romanesque qui réconcilie l'oralité des contes de Zohra et la raison graphique de la littérature française (ou francophone) à laquelle elle s'agrège.

L'homologie rite/récit qu'étudie l'ethnocritique permet de mesurer la dimension subversive de l'écrit dans un ordre social coercitif et rétrograde. 


\title{
Homologie libération nationale/émancipation des filles par l'instruction publique
}

18 l'Algérie. La guerre commence " un mois après son entrée à l'école »; le texte établit une sorte de pacte narratif et historique : la liberté de la nation algérienne ne peut aller de pair qu'avec l'émancipation des filles par l'instruction. Fascinée par les djounouds (les combattants qui prennent le maquis), elle reçoit cette injonction révolutionnaire: «Ton maquis à toi, c'est l'école!» (ibid., p. 202). La directrice de l'école, une Française communiste favorable à l'indépendance, réussit à convaincre le père de Leïla de la laisser passer en $6^{\mathrm{e}}$ en invoquant le sens profond de la révolution : « la scolarisation des enfants est l'une des priorités du combat pour la liberté »(ibid., p. 214). Or les espérances d'une nation indépendante vont montrer des résultats contraires, renforcement des inégalités hommes/femmes, séparation ethnique entre Arabes et Berbères, arabisation et islamisation de l'école :

Amère indépendance que celle qui livrait des fournées entières d'enfants à des fascistes se servant du Coran, merveille littéraire, pour tuer la langue arabe dès l'école primaire. Car si les élèves ânonnaient par cœur les versets du Coran, ils méconnaissaient cette langue. (ibid., p. 302)

«L'écrit oralisé » remplace la littératie, une langue méconnue remplace la langue française, la récitation remplace «l'esprit critique» (ibid., p. 302). La révolution algérienne apparaît, au miroir des hommes qui marchent d'un côté, et de l'instruction de Leïla de l'autre côté, non pas comme un retour à l'ordre traditionnel - encore moins comme un progrès -, mais comme un enfermement dans des modèles exogènes destructeurs : l'islamisme des Frères musulmans égyptiens (ibid., p. 283) et le soviétisme russe (ibid., p. 312). Le coup d'État de H. Boumédiène et la mise en place d'une «brigade des mœurs ", marquent la fin de l'espérance de la Houria (ibid., p. 314).

\section{Une écriture métissée : la rencontre des mondes}

\author{
Le bracelet est arc-être \\ aujourd'hui demain hier \\ (Hawad, Caravane de la soif)
}

Refusant les oppositions binaires qui ont ruiné la révolution algérienne, le texte opte pour une articulation ternaire: trois figures féminines servent de modèle à Leïla (Saâdia, Madame Bensoussan l'institutrice et la Bernard, la sage-femme ; ibid., p. 133); parallèlement, elle reconnaît trois héritages qui sont autant de moyens de résistance (le "démon de Bouhaloufa", Zohra et ses "métaphores", l'école et la littérature françaises; ibid., p. 141) ; enfin elle identifie trois «mondes» auxquels elle appartient en partie tout en y demeurant toujours marginale. Lors du deuxième passage des Touaregs venus célébrer l'indépendance à Kenadsa, Leïla distingue ces trois mondes : « celui des humanistes roumis incarnés par Portalès, celui des citadins, les Bouhaloufa et ses parents. Celui enfin des hommes bleus et de Zohra " (ibid., p. 237). Cette réunion est une image de ce qu'aurait pu, et dû être la Houria, à savoir une intelligence entre les "communautés" (Européens, Berbères et Arabes) et les religions (islam, judaïsme, christianisme), mais surtout une rencontre des mondes qui traversent le personnage principal et l'écriture du livre. Qu'est-ce qu'un «monde» pour le texte? L'horizon 
"descriptif et interprétatif» de l'ethnocritique est «la culture du texte dans sa singularité et non l'indexation de la culture du texte..$^{28}$ » Le roman propose un concept, celui de monde, qui va structurer la composition du texte. Un monde ne se confond pas avec une ethnie ou une communauté (catégories extérieures au textes); ainsi un monde peut diviser une communauté : Portalès est un Pied-noir comme les sont les parents de Gisèle qui insultent Leïla, mais son humanisme le sépare de leur "monde ». De même, un «monde » peut réunir des communautés séparées : Zohra, «la bédouine » (ibid., p.10) arabophone, appartient au même monde que les Berbères que l'on appelle les « hommes bleus " parce qu'ils partagent une même cosmologie nomade. Le personnage de Leïla se considère comme métisse ; elle revendique un lointain métissage ethnique en valorisant, au grand dam de sa mère, la «filiation maudite» (ibid., p. 206) venue d'une aïeule noire : «Une goutte d'ébène qui épanouissait une lippe nègre au milieu d'un visage aux traits de pur maure, coiffait ici une tête d'une crinière crépue au milieu d'autres aux cheveux souples. Leilla aimait ça!» (ibid., p. 206). Or c'est davantage un métissage culturel qui la caractérise, ce qui suscite une joie douloureuse: "Pétrie de pâtes si différentes, ne deviendrait-elle pas une métisse qui serait, un jour peut-être, reniée par tous?» (ibid., p. 238). La richesse du personnage qui garantit son émancipation la condamne à la solitude.

C'est à l'écriture de porter les espérances trahies de la Houria, de questionner une société qui a rejeté la pluralité culturelle. Le personnage de Leïla est à l'image de l'écriture du texte : un amalgame qui métamorphose la rencontre de plusieurs cultures en une culture nouvelle, créative et dynamique. Ce type de métissage, extrêmement valorisé pour les Touaregs, porte le nom de tikruru; les tikruruten sont « ceux qui font le lien entre les peuples, comme par exemple les grands commerçants, les grands caravaniers qui relient le nord au sud, les lettrés musulmans, les médecins qui associent les connaissances de la Méditerranée, de l'Afrique noire et de l'Orient. ${ }^{29}$ » Ici, l'ethnocritique nous permet de reculturer ${ }^{30}$ le texte en éclairant le refoulé berbère saharien qui imprègne la culture du texte : le paradoxe est que le dépassement des dichotomies forgées par les idéologies coloniales et nationalistes aboutit finalement au retour de la cosmologie non pas "archaïque » mais proprement indigène au Sahara (plus qu'à l'Algérie), toujours vivante parmi les Touaregs dans leur langue (le tamasheq), et toujours à l'état de latence chez les arabophones. L'inquiétude identitaire est celle d'une écriture marquée par les déchirures du xxe siècle ; c'est aussi le fait d'une conception du monde qui doit équilibrer la diversité avec le métissage : un monde sans tikruru serait un monde sans échanges culturels, mais à l'inverse, si les cultures venaient à se fondre en une seule, il n'y aurait plus l'extériorité (l'essuf en tamasheq, qui désigne à la fois la solitude et l'espace étranger, dangereux, que l'on doit rencontrer dans chaque rite de passage) et par conséquent il n'y aurait «plus de seuil à franchir, plus d'initiation possible »: "sans altérité, pas de progression et sans symbiose, pas d'ascension ${ }^{31} »$.

Cette remarque nous permet de comprendre que la «progression » sociale de Leïla est conditionnée par l'altérité que représente sa grand-mère et les Européens qu'elle fréquente à l'école ; une société sans altérité est donc logiquement stagnante. De même, son agrégation finale avec le statut d'écrivain est une véritable "ascension » : à son tour elle devient passeur, taggagt, du fait de la "symbiose " qu'elle opère - dans l'écriture -, entre l'oralité bédouine de Zohra, la culture arabe écrite de Bouhaloufa et la littérature française : «Sa plume se mit à écrire avec fébrilité, comme sous la dictée de 
l'aïeule qui revivait en elle » (ibid., p. 321). Le roman s'achève là où il avait commencé ; comment comprendre cette architecture textuelle sans angles, qui dessine une courbe à l'instar des bracelets touaregs? Dans la cosmologie touarègue, ce retour au point de départ n'est pas un échec, mais, au contraire, un accomplissement cosmique : "les anneaux d'argent des femmes touarègues symbolisent le parcours nomade cyclique dont l'achèvement annonce un nouveau départ ${ }^{32} »$. Il ne s'agit plus seulement d'un rite de passage pour l'homme social, mais pour l'homme cosmique : «Dans ce schéma, la fin du cycle est est le début d'un nouveau cycle, c'est pourquoi psychologiquement, lorsque la boucle est bouclée, le marcheur a le sentiment de se retrouver à son point de départ ${ }^{33}$ ", c'est-à-dire au tamejirt, le campement laissé en arrière. De retour au pays natal, retour qui transfigure la jeune révoltée en écrivaine, Leïla délaisse la maison de pierres de ses parents (dâr) pour se retrouver sur la dune qu'elle a toujours considérée comme sa réelle domus, ou plutôt son tamejirt. La poétique du texte ne se pense pas en termes d'architecture du texte : nous parlerions plutôt de marche du texte comme de l'identité. Ainsi, le motif culturel qui explique cette marche circulaire est l'Inta, notion centrale de la cosmologie touarègue que le poète $\mathrm{M}$. Hawad définit comme «l'étape de l'harmonie et de l'unité absolue ; c'est le premier pas dans le dernier, l'instant d'équilibre qui suit la fin d'une action et précède le début d'une autre ${ }^{34} »$.

\title{
Écriture, discours méta-critique, engagement politique : quelle place pour la critique?
}

\author{
Pas même musicien \\ Je n'entends que l'effacement des sons \\ Pas même écrivain \\ Je trace seulement les lignes du silence
}

(Hawad, 1985) et Arabes, dont la cosmologie échappe aux représentations graphiques héritées de la colonisation. Le texte fait revivre une forme de pensée et de communication qui n'a plus cours, et que la Révolution algérienne a trahie, au nom de fausses racines puisées dans l'arabo-islamisme. Ce roman pleinement engagé tient un discours sans ambiguïté sur le colonialisme, sur le système de coercition phallocrate ayant cours en Algérie, sur le racisme et l'antisémitisme dominants au Maghreb, sur la montée de l'islamisme organisée par l'arabisation forcée. Par ailleurs, l'auteure réhabilite l'école française, et plaide pour l'instruction obligatoire, pour le développement de la culture du livre: après avoir opéré une fuite-en-arrière par la mise en scènes des oralités pré-coloniales, le roman opte pour une fuite-en-avant dans la promotion pour la littératie, instrument d'égalité hommes-femmes et d'émancipation individuelle et collective. dépasser le discours de l'auteure, pour analyser le travail de l'écriture. La "poétique culturelle du texte ${ }^{35}$ » est, dans Les Hommes qui marchent, un métissage des cosmologies selon le modèle du tikruru : l'écriture est travaillée par une belligérance et un dialogue entre oralité primaire (véritable oralité de la parole de Zohra, figure de griote maure ou de taggagt touarègue), oralité secondaire (auralité caractéristique de la société clanique) et raison graphique (entrée dans le monde régi par la littératie, l'alphabet latin et la langue des écrivains d'expression française). Le roman reconfigure un univers singulier, 
composé de plusieurs "milieux» ou "mondes» sociaux et culturels en pleine décomposition et re-composition, suite aux tragédies de l'Histoire. Sans cette création d'un monde, le livre ne serait qu'un ensemble de chroniques amères oscillant entre la déploration stérile d'un passé révolu et la critique acerbe des échecs de la révolution: ce ne serait pas un roman mais un récit autobiographique agrémenté de descriptions du désert, ou une série de "vies minuscules", fragments de vies des personnages (histoire de Zohra, histoire de Djelloul, etc.). Il y avait donc matière à produire au moins trois livres, mais Malika Mokeddem a fait le choix du roman, de la «philosophie du roman $^{36}$ " avec ses conflits de cosmologies, le choix d'une écriture portée par une forte dimension autoréflexive. Nous avons voulu démontrer que les schèmes issus des cultures maghrébines et sahariennes pré-coloniales et pré-nationales structurent en profondeur la création verbale et narrative chez Malika Mokeddem. Le discours militant et critique fait de l'auteure une figure d'intellectuelle majeure pour le $\mathrm{xxl}^{\mathrm{e}}$ siècle en proie aux «identités meurtrières ${ }^{37}$ »; parallèlement, son travail d'écrivain sur une langue française traversée par les oralités maures et berbères autant que par des littératies arabes et françaises, est plus riche et plus utile encore pour un siècle qui n'est pas guéri du malaise dans la culture post-coloniale.

Nous voudrions ouvrir une nouvelle voie de la critique : à partir de l'anthropologie sur les cultures du Sahara (H. Claudot-Hawad, J. Drouin et C. Taine-Cheikh), nous pourrions mettre en lumière une logique culturelle particulière, liée à un espace géographique, le Sahara, travaillant en profondeur l'écriture d'écrivains installés de part et d'autre de la Méditerranée, ou du Sahara. Cette logique qui traverse l'écriture de Malika Mokeddem transcende les clivages nationaux (Algérie/Mali/Niger), les oppositions ethniques (Berbères/Maures/Arabes), les communautés religieuses (Juifs/Chrétiens/Musulmans), mais aussi la division entre Afrique Blanche et Afrique Noire. Ce travail se veut donc une proposition ouvrant vers d'autres recherches.

\section{BIBLIOGRAPHIE}

BOURDIEU, P. (1987). Choses dites. Paris : Éditions de Minuit.

BOUVET, R. (2019). Tisser les Voix. Montréal : Mémoire d'encrier.

CLAUDOT-HAWAD, H. (1996). « Touaregs : l'identité en marche », Études et Documents berbères 14, p. 223-232.

CLAUDOT-HAWAD, H. (2002). Touaregs. Apprivoiser le désert. Paris : Gallimard.

CLAUDOT-HAWAD, H. (2006). « Marginale l'étude des marges. Parcours en terrain touareg In :

ClAUdOT-HAWAD, H. (dir.). Berbères ou Arabes? Le tango des spécialistes. Paris/Aix-en-Provence : Éditions Non Lieu/Institut de Recherches et d'Études sur le Monde Arabe et Musulman, p. 191-223. 
CNOCKAERT, V. \& DUMOULIN, S. (2015) « Oralités/Littératies/Littératures ». In : David, A.-M. \& Popovic, P. (dirs). Les douze travaux du texte. Sociocritique et ethnocritique. Montréal : Université du Québec à Montréal, p. 121-130.

DESCOMBES, V. (1987). Proust. Philosophie du roman. Paris : Éditions de Minuit.

DROUIN, J. (1987) « De quelques conceptions de la parole dans la société touarègue ». Journal des Africanistes 57, fascicule 1-2, p. 77-96.

FABRE, D. (2008) « Chinoiserie des Lumières. Variations sur l'individu-monde », L'Homme 185-186, p. 269-299.

Golovanov, V. (2012) [2008]. Espace et labyrinthes. Trad. du russe par H. Châtelin. Lagrasse :

Verdier.

GOODY, J. (1978) [1977]. La Raison graphique. La domestication de la pensée sauvage. Trad de l'anlgais par J. Bazin \& A. Bensa. Paris : Éditions de Minuit.

HAWAD, M. (1985). Caravane de la Soif. Trad. du touareg par H. Claudot. Aix-en-Provence, Edisud, 1985.

LÉVI-STRAusS, C. (2008) [1962]. « La Pensée sauvage ». In : Cuvres. Paris :Gallimard.

LÉVI-STRAuss, C. (1973). « La Geste d'Asdiwal ». In : Anthropologie structurale, 2. Paris : Plon, p. 175-233.

MAALOUF, A. (2001) [1998]. Les Identités meurtrières. Paris : Librairie générale française.

MOKEDDEM, M. (1990). Les Hommes qui marchent. Paris : Éditions Ramsay.

PRIVAT, J.-M. (2006). « Un habitus littératien? », Pratiques 131-132, p. 125-130. En ligne : https:// www.persee.fr/doc/prati_0338-2389_2006_num_131_1_2122.

PRIVAT, J.-M. (2018). « Sur La Raison graphique. La domestication de la pensée sauvage de Jack Goody », Questions de Communication 33, p. 299-323.

PRIVAT, J.-M. (2015). « Ethnocritique. Signes de naissances ». In : David, A.-M. \& Popovic, P. (dirs). Les douze travaux du texte. Sociocritique et ethnocritique. Montréal : Université du Québec à Montréal, p. 27-42.

PRIVAT, J.-M. \& Scarpa, M. (2013). « Ethnocritique et anthropologie(s) des littératures ». L'Homme 206, pp. 183-190.

SCARPA, M. (2013). « De l'ethnologie de la littérature à l'ethnocritique ». Recherches \& Travaux 82, p. 21-27.

SCHAEFFER, J.-M. (1995) « Littérature orale ». In : Ducrot, O. \& Schaeffer, J.-M. Nouveau dictionnaire encyclopédique des sciences du langage. Paris : Seuil.

TAINE-CHEIKH, C. (2014). « Langues, savoirs et pouvoirs en milieu maure ». In : Bonte, P. \& CLAUDOTHAWAD, H. (dirs). Élites du monde touareg et maure. Aix-en-Provence : Institut de recherches et d'études sur les mondes arabes et musulmans, p. 167-184. En ligne : https:// books.openedition.org/iremam/2671?\#text. VAN GENNEP, A. (2004) [1909]. Les Rites de Passage, Paris : Picard.

VIDAL-NAQUET, P. (2005) [1981]. Le Chasseur noir. Formes de pensées et formes de société dans le monde grec, Paris : La Découverte. 


\section{NOTES}

1. J.-M. Privat définit la littératie comme «l'ensemble des praxis et des représentations liées à l'écrit, depuis les conditions matérielles de sa réalisation effective (support et outils techniques d'inscription) jusqu'aux objets intellectuels de sa production et aux habiletés cognitives et culturelles de sa réception, sans oublier les agents et institutions de sa conservation et de sa transmission " (cité dans Cnockaert \& Dumoulin, 2015, p. 125).

2. Fabre, 2008, p. 275.

3. Scarpa, 2013, p. 26.

4. Scarpa, 2013, p.27.

5. H. Claudot-Hawad montre que c'est dans les années 30 que se construit un discours sur "l'identité algérienne ", influencé par deux apports étrangers, la oumma islamique et le jacobinisme français. De là l'élaboration de «l'arabo-islamisme comme trait définitoire et exclusif de l'identité algérienne » (Claudot-Hawad, 2006, p. 211-212).

6. J'emprunte cette idée à H. Claudot-Hawad (1996, p. 223-224) pour qui les notions d' identités et d'ethnies sont des "catégories négociées » : «Dans la cosmologie touarègue, tout être, toute chose, tout élément apparaît mobile, engagé dans une succession de cycles qui reproduisent le mouvement de l'univers".

7. «L'attrait des Touaregs pour les vêtements teints à l'indigo et le bleuissement de la peau qui en résulte leur a valu, de la part des étrangers, le surnom d'hommes bleus » (Claudot-Hawad, 2002, p. 47).

8. J'emprunte la formule à R. Bouvet (2019).

9. Claudot-Hawad, 2002, p. 18.

10. Taine-Cheikh, 2014.

11. Cnockaert \& Dumoulin, 2015, p. 122.

12. Schaeffer, 1995, p. 515.

13. Taine-Cheikh, 2014.

14. Nous reprenons les catégories de C. Lévi-Strauss (1973, p. 175-233) qui dans «La Geste d'Asdiwal ", démontre qu'un mythe se compose, sur un axe syntagmatique, de "séquences", et sur un axe paradigmatique de quatre "schèmes" ou "niveaux": géographique, social, techno-économique et cosmologique.

15. Sur le prix accordé à la « puissance des talismans » dans l'Islam et le Judaïsme, voir la lettre de Rica à Nathanaël Lévi dans les Lettres persanes de Montesquieu (lettre 143).

16. Taine-Cheikh, 2014.

17. Claudot-Hawad, 2002, p. 45.

18. «De quelques conceptions de la parole dans la société touarègue » (Drouin, 1987, p. 80).

19. H. Claudot-Hawad, 1996, p. 226.

20. Je reprends le titre de V. Golovanov (2012 [2008]).

21. Jean-Marie Privat, 2018.

22. J. Goody (1978 [1977], p.166) rappelle à ce propos que Hérodote considérait les Égyptiens comme les plus savants car ils tenaient scrupuleusement leurs archives. 
23. Lévi-Strauss, 2008 [1962], p. 811. Au chapitre XI « Histoire et dialectique ", l'auteur résume : «Le propre de la pensée sauvage est d'être intemporelle; elle veut saisir le monde, à la fois comme totalité synchronique et diachronique » (ibid., p. 841).

24. Lévi-Strauss, 2008 [1962], p. 811.

25. Van Gennep, 1998.

26. Bourdieu, 1987, p. 132-143.

27. Privat, 2006.

28. Privat \& Scarpa, 2013, p. 187.

29. Claudot-Hawad, 1996, p. 229.

30. M. Scarpa (2013, p. 26.) écrit que « le rapport à l'œuvre doit être reculturé ».

31. Claudot-Hawad, 1996, p. 232.

32. Claudot-Hawad, 1996, p. 53.

33. Claudot-Hawad, 1996, p. 226.

34. Hawad, 1985, p. 19.

35. Privat, 2015, p.38.

36. Descombes, 1987.

37. Maalouf, 2001, [1998].

\section{RÉSUMÉS}

Cet article présente une analyse du roman Les Hommes qui marchent; celle-ci s'appuiera sur l'hypothèse de l'ethnocritique qui envisage une homologie entre rite et récit. La scolarisation du personnage principal est envisagée comme un rite de passage qui la conduit à un avenir émancipé dans le contexte de la guerre d'Algérie puis de la Révolution algérienne. L'écriture et l' habitus littératien se veut une révolte contre toutes les formes de coercitions qui assignent les individus (en particulier les femmes) à une place prédéfinie et inamovible. L'écriture renoue alors avec le nomadisme des aïeux maures sahariens, avec un monde antérieur aux découpages géographiques et ethniques issus de la colonisation et des États post-coloniaux. Cette étude montre comment une pensée authentiquement saharienne, nomade, pénètre en profondeur une écriture romanesque qui devient, à l'image de l'espace saharien, un espace de rencontre entre les langues et les cosmologies, entre les oralités et les littératies.

This essay analyzes Malika Mokeddem's novel Les Hommes qui marchent following the assumption that there is, as seen in other ethnocritic researches, an homology between rite and tale. The main character's schooling is seen as a rite of passage that leads to her emancipation during the war of Algeria and the Algerian revolution. In this particular case, literacy serves a project against all forms of coercion which assigns people (specially women) to a predetermined and unmovable position. The act of writing reconnects with the nomadism of her Saharan Moors ancestors and with a world that was erased by the new geographical and ethnic divisions that came with the colonization and then the independence. This case study shows how an authentically Saharan and nomad thinking irrigates deeply a fiction writing that is meant to 
become, just like the Sahara Desert, a meeting place between languages and cosmologies, between oralities and literacies.

\section{INDEX}

Mots-clés : ethnocritique, homologie rite/récit, oralités/littératie, nomadisme/sédentarité, Sahara

Keywords : Ethnocriticism, rite/story homology, oralities/literacies, nomadism/sedentary lifestyle, Sahara

\section{AUTEUR}

\section{SIMON LANOT}

Université de Lorraine, Crem, F-57000 Metz, France 